\title{
Mouvement associatif des femmes en Mongolie et partenariats internationaux
}

Exemple d'un « contre-effet boomerang "

Women's associations movement in Mongolia and international partners: a case

of « counter boomerang effect"

\section{Anna Jarry-Omarova}

\section{(2) OpenEdition}

Journals

\section{Édition électronique}

URL : http://journals.openedition.org/conflits/18201

DOI : $10.4000 /$ conflits. 18201

ISSN : $1777-5345$

Éditeur :

CCLS - Centre d'études sur les conflits lilberté et sécurité, L'Harmattan

\section{Édition imprimée}

Date de publication : 30 décembre 2011

Pagination : 79-98

ISBN : 978-2-296-55700-0

ISSN : 1157-996X

Référence électronique

Anna Jarry-Omarova, « Mouvement associatif des femmes en Mongolie et partenariats

internationaux », Cultures \& Conflits [En ligne], 83 | Automne 2011, mis en ligne le 04 janvier 2013,

consulté le 30 mars 2021. URL : http://journals.openedition.org/conflits/18201 ; DOI : https://doi.org/ 10.4000/conflits. 18201 


\title{
Mouvement associatif des femmes en Mongolie et partenariats internationaux
}

\author{
Exemple d'un " contre-effet boomerang "
}

\section{Anna JARRY-OMAROVA}

Anna Jarry-Omarova est docteure en sociologie politique de l'EHESS, Paris. Sa thèse a analysé différentes dynamiques sociétales qui permettent d'expliquer l'échec $d u$ mouvement associatif des femmes en Mongolie depuis la démocratisation $d u$ pays en 1992. Elle a enseigné la socio-anthropologie du genre à l'université ParisDiderot, rattachée au Centre d'Etudes, de Documentation et de Recherches en Etudes Féministes (CEDREF). Ses recherches portent aujourd'bui sur "la globalisation du genre " et son utilisation par les associations de femmes en Asie centrale.

$\mathrm{E}$ n 1992 et à la suite de soixante-dix ans de régime communiste, la Mongolie a adopté une Constitution démocratique dont les dispositions législatives en font, selon les observateurs internationaux, « le pays le plus libéral du monde ${ }^{1}$ ». Ce sont les jeunes intellectuel(le)s ayant suivi des études supérieures en Europe de l'Est et à Moscou à la fin des années 1980 qui, en revenant au pays pour prendre leurs postes de hauts fonctionnaires, ont enclenché le processus de transition, immédiatement suivis par la population, urbaine comme rurale. Ces jeunes activistes sont sans distinction des jeunes hommes et des jeunes femmes, qui participent ensemble à l'établissement des nouveaux partis politiques d'opposition démocrate ${ }^{2}$. En parallèle, les femmes investissent et organisent le nouvel espace public qui s'ouvre à elles, celui des associations. Comme cela avait été le cas dans le cadre du mouvement démocratique, ces initiatrices et dirigeantes d'associations sont immédiatement rejointes par de très nombreuses militantes urbaines mais aussi rurales, de toutes les catégories sociales : fonctionnaires locales, commerçantes, artistes, etc. Les femmes mongoles sont en effet rompues à ce type d'activités civiques puisqu'elles en menaient déjà au sein du Comité national des femmes, l'ancien

1. «En avril 1997, le Parlement a décidé de supprimer la plupart des taxes d'import et d'export, ce qui procure à la Mongolie l'un des régimes les plus libéraux du monde " (Ginsburg T., "Mongolia in 1997: Deepening Democracy", Asian Survey, vol.38, n¹, 1998, p. 66).

2. Pour une analyse de la constitution de l'espace politique démocratique en Mongolie, voir la première partie de notre thèse de doctorat : http://tel.archivesouvertes.fr/docs/00/54/57/81/PDF/these_Jarry-Omarova_2010.pdf 
organe féminin du parti communiste (le Parti populaire révolutionnaire mongol, PPRM). De fait, on peut dire qu'au lendemain de la démocratisation, la société civile en Mongolie s'est exclusivement composée d'associations de femmes. S. M. Fish, un des nombreux observateurs américains sur place dans les années 1990, déclarait ainsi : " la plus formidable composante de la société civile se trouve être les associations de femmes. Ces groupes sont si forts et les femmes sont si dominantes dans tous les types d'organisations civiques, qu'on pourrait presque parler de société civile matriarcale en Mongolie 3 ».

Un second point caractérise ce mouvement des femmes en Mongolie : il ne s'est pas constitué autour de la lutte contre la pauvreté ou pour le contrôle de la fécondité par exemple, thématiques communes des mouvements de femmes dans le monde. L’objectif premier et déterminé de ces associations de femmes - et ce, dès 1992 - est la participation à la construction de cette nouvelle démocratie en laquelle elles ont placé tous leurs espoirs, la défense de «l'égalité de genre » (жендерийн тегш эрх, genderijn teǧ̌ erh, c'est-à-dire mot à mot «droits de l'égalité de genre ») et la promotion des femmes en politique. Elles visent donc immédiatement " haut », à savoir l'espace du pouvoir dont elles sont écartées. Ces dernières constatent en effet que dès la mise en place d'une Assemblée constituante en 1990-1992, seuls neuf des 462 représentants, sont des femmes, soit à peine $2 \%$. Elles vont alors déployer une énergie remarquable dans leur lutte : conférences, articles de presse, rencontres au sein des partis politiques 4, construction d'un savoir sur les rapports hommes/femmes dans le pays avec les premières enquêtes sur ces questions, interventions universitaires, etc. Elles organisent surtout une Coalition des femmes pour soutenir les candidates lors de la campagne électorale parlementaire du printemps 2000, tous partis confondus. Les représentantes s'invitent aux conseils de partis, à tous les meetings publics et même au Parlement, munies de rapports sur cette question de l'égalité entre hommes et femmes. Elles y prennent la parole, accompagnées des députées, créant des sortes de happenings politiques relayés par la presse et la télévision ${ }^{5}$. Pourtant, et mal-

3. Fish M. S., “Mongolia: Democracy without prerequisites”, Journal of Democracy, vol.9, n³, 1998, p. 137.

4. Le paysage politique se divise, pendant cette première décennie démocratique, entre le PPRM (anciens communistes constituant une "gauche ») et les deux partis démocrates : le Parti social-démocrate et le Parti national-démocrate. Les communistes vont gagner les élections en 1992, une coalition démocrate en 1996, puis les communistes reviennent en 2000 jusqu'à aujourd'hui. Le pays se dote d'une caractéristique : une cohabitation récurrente entre un parlement d'une majorité politique et son président de la République, élu l'année suivante dans le cycle électoral, de la tendance opposée. Ainsi, le premier président fut démocrate avec un parlement communiste, et c'est de nouveau le cas depuis 2009. De 1997 à 2001, le parlement était démocrate et le président communiste. Ce n'est qu'entre 2001 et 2005 que le pays a été dirigé par un gouvernement et un président communiste, Enkhbayar, que nous avons d'ailleurs rencontré lorsqu'il était encore secrétaire général du Parti. Une multitude de petits partis va ensuite émerger à la faveur de la crise des démocrates à partir de 1998, sans qu'aucun ne réussisse à vraiment redessiner le paysage composé des deux tendances majeures.

5. Pour une présentation générale du mouvement associatif, voir la troisième partie de notre thèse de doctorat : http://tel.archives-ouvertes.fr/docs/00/54/57/81/PDF/these_JarryOmarova_2010.pdf ; voir également Jarry-Omarova A., «Sphère publique, sphère politique : 
gré tout ce travail, le nombre de femmes députées n'augmente pas. En 2000, soit près d'une décennie après la transition (et trois élections démocratiques), elles ne sont à nouveau que huit députées sur 76 au total, le même nombre qu'en 1992 et 1996. En 2008, seules trois députées sont des femmes (soit 4 \%). Elles n'ont pas plus de succès lors de la formation du gouvernement qui ne compte aujourd'hui que trois femmes ministres et deux secrétaires d'État. Et alors qu'elles représentent $24,6 \%$ des conseillers régionaux, aucune n’a été nommée gouverneure de région depuis 20006 .

Constatant une « hyper activité » du mouvement des femmes en Mongolie et s'interrogeant sur son échec, paradoxal à première vue, nous avons exploré plusieurs hypothèses. L'étude des obstacles intrinsèques au système démocratique a montré par exemple la dynamique d'élimination des candidates au sein des partis politiques. De même, l'analyse de l'idéologie de genre de la culture nomade patriarcale a permis de comprendre son impact sur la société mongole actuelle. Cependant, l'hypothèse principale de notre travail nous a été suggérée par les militantes elles-mêmes, lorsqu'elles disaient invariablement lors des entretiens : « Notre plus gros problème ? C’est le manque d'argent ! 7 ». En effet, l'État n'apporte aucun financement à leurs activités car ce n'est pas prévu dans les politiques sociales qui sont d'ailleurs réduites à peau de chagrin depuis 1991, principalement en raison des injonctions du Fonds monétaire international (FMI). Quant au mode bien connu en Europe du financement par cotisations individuelles, il ne saurait en être question : la libéralisation de l'économie a considérablement appauvri la population. Les associations de femmes se sont alors tournées vers les seules entités qui, non seulement, proposaient des financements mais, en outre, semblaient très intéressées par leur lutte : les organisations internationales de développement (étatiques, mais aussi fondations privées et religieuses) et leurs programmes de développement ciblés « genre ». Se saisir de cette nouvelle notion de « genre » était d'ailleurs une démarche incontournable pour obtenir leurs subventions. Comme exposé en introduction du présent numéro, les études féministes ont largement mis à jour les effets pervers des partenariats entre mouvements locaux de femmes et directives internationales, notamment économiques ${ }^{8}$. Nous ne reviendrons

le cas des associations de femmes en Mongolie », L'Homme et la société, n¹58, 2005. En ligne : http://www.cairn.info/resume.php?ID_ARTICLE=LHS_158_0201; Jarry-Omarova A., «Une association à la croisée de différents espaces : le cas de CEDAW Watch en Mongolie. ", in Tahon M-B. (ed.), Les femmes entre la ville et la cité, actes du 4e Congrès international des recherches féministes dans la francophonie plurielle, Ottawa/Montréal, Le Remue-Ménage, 2007.

6. Rapport Comité CEDEF, 2008, CEDAW/C/MNG/Q/7/Add.1.

7. Les entretiens cités ont été réalisés entre 1999 et 2001, en anglais et en mongol. La traduction en français a été effectuée par nos soins. Précisons que personne n’a jamais demandé l'anonymat (que nous préservons cependant selon les situations et lorsqu'il ne s'agit pas de personnalités publiques) et qu'en Mongolie, les personnes sont nommées par leur prénom (accompagné de la majuscule de leur patronyme au besoin), le président de la République y compris.

8. Citons en particulier Bisilliat J., Regards de femmes sur la globalisation : approches critiques, Paris, Karthala, 2003 ; Falquet J., De gré ou de force. Les femmes dans la mondialisation, Paris, La Dispute, 2008 ; Hours B., Selim M., Anthropologie politique de la globalisation, Paris, 
pas sur cette thématique ici, sauf à dire que les programmes de développement en Mongolie se prêtent également à ce type d'analyse critique 9 . Cette question des moyens matériels, en revanche, demeure pertinente en ce qu'elle a permis l'entrée en jeu d'entités étrangères, et nous mène vers une analyse des interactions au sein d'un triptyque d'acteurs : le mouvement associatif des femmes (1), qui tente d'agir sur l'État et ses officiants au gouvernement (2), et qui n'est financé que par ses partenaires internationaux (3). Si les militantes ont pu observer un semblant d' "effet boomerang » au début des années 1990 tel que décrit par Keck et Sikkink ${ }^{10}$, c'est-à-dire le contournement de l'obstacle - l'État - pour faire pression sur celui-ci et obtenir in fine des avancées législatives, cette dynamique a immédiatement été « interceptée », et c'est bien plutôt un «contre-effet boomerang » auquel nous avons assisté en Mongolie à la fin des années 1990. Et ce, avec pour argument principal la protection de la nation.

\section{Effet boomerang}

Avec l'acquisition de nouveaux droits et libertés, et notamment l'accès à la propriété privée, la seconde dimension majeure de la démocratisation fut l'ouverture à l'international. La Mongolie et son peuple nomade ont une longue histoire de relations avec des étrangers ${ }^{11}$ et, sous le régime communiste, les échanges étaient denses au sein du bloc soviétique (échanges sportifs, intellectuels, artistiques, professionnels et politiques avec les capitales communistes, de Berlin-Est à Hanoi). La population n'était pourtant pas satisfaite de ne pouvoir sortir de cette zone de «similitude ». Dorénavant, l'article 18 de la nouvelle Constitution énonce la liberté de se déplacer à l'intérieur du pays, d'une Région administrative à une autre (auparavant, il fallait une autorisation des autorités locales), de voyager et de résider à l'étranger. La parlementaire démocrate Narangerel le met en avant : «Le point positif principal en Mongolie aujourd'hui ? C'est le fait que je peux parler avec vous, maintenant. La liberté, vous voyez ? La liberté 12 ». Ce qu'apporte la démocratisation, ce sont de nouvelles relations diplomatiques, culturelles et commerciales avec les

L'Harmattan, 2010 ; Peemans Poullet H., « La miniaturisation de l'endettement des pays pauvres passe par les femmes... ", Chronique féministe, n71-72, février-mai 2000 ; Treillet S., "L'enrôlement des femmes dans la marchandisation libérale », L'Humanité des débats, 10 octobre 2007 ; Verschuur C. (ed.), Genre, postcolonialisme et diversité des mouvements de femmes, Paris, L'Harmattan, 2010.

9. Voir en particulier le chapitre 7 de notre thèse de doctorat, qui apporte encore des exemples de programmes de développement ciblés « genre » qui renforcent pourtant la domination masculine.

10. Keck M. E., Sikkink K., Activists beyond Borders: Advocacy Networks in International Politics, Ithaca et Londres, Cornell University Press, 1998.

11. Voir à ce sujet le témoignage de G. de Rubrouck (Kappler C., Kappler R., Voyage dans l'empire mongol: 1253-1255, Paris, Imprimerie nationale, 1997,) qui rencontre à la cour du Khan au XIII siècle des nestoriens, des chrétiens et des Sarrasins (p. 109) et même " une Lorraine de Metz qui avait été faite prisonnière en Hongrie ", mariée à un jeune Ruthène [Europe du nord-est, Ukraine], dont elle avait trois jeunes enfants très beaux (p. 149).

12. Députée du Parti social-démocrate, rencontrée à Oulan-Bator le 29 juillet 1999. 

nouveaux partenaires est général et il en est de même pour les associations de femmes qui lancent toutes, sans exception, des projets avec des organisations internationales. En 2000, nous pouvions lister l'Agence pour les Nations Unies, la Banque mondiale, l'USAid 13, l'Asia Foundation ${ }^{14}$, le Global Found for Women 15, l'Open Society Institute (fondation du milliardaire G. Soros ${ }^{16}$ ), ou encore le Canada Found. Sont aussi présents le programme européen TACIS ${ }^{17}$, la Konrad Adenauer Foundation du gouvernement allemand, une organisation hollandaise de défense de l'environnement, des associations caritatives japonaises ${ }^{18}$, etc. Même l'organe féminin du traditionnel parti communiste, l'Union des femmes démocrates et socialistes au sein du PPRM a trois programmes financés par le gouvernement hollandais, le Programme des Nations Unis pour le développement (PNUD) et le Fonds des Nations unies pour l'enfance (UNICEF) ${ }^{19}$. Les financements sont variables et peuvent atteindre 50000 US\$ (soit environ $37000 €$ quand un salaire moyen en 1999 est de $40 €$ par mois, jusqu'à $80-100 €$ pour des postes de cadres). Ces organisations internationales constituent une véritable ressource matérielle et, à la fin des années 1990, toutes les associations, sans exception, y sont liées.

13. Agence gouvernementale étatsunienne, chargée du développement économique et de l'assistance humanitaire dans le monde.

14. ONG internationale dont la maison mère se trouve à Washington DC. Les 87 millions de US\$ de budget pour l'année 2008 et les 41 millions de US\$ pour des publications « éducatives » proviennent de nombreux donateurs, en particulier le gouvernement américain : http://asiafoundation.org/about/

15. ONG dont le siège est à San Francisco, financée par la Banque mondiale et de nombreuses églises et congrégations catholiques : http://www.globalfundforwomen.org/cms/aboutgfw/supporters/

16. Pour une analyse critique de son impact dans le monde post-soviétique, voir les travaux de Guilhot N., "La promotion internationale de la démocratie : un regard critique ", Mouvements, n`18, 2001, pp. 28-31; et "Une vocation philanthropique ", Actes de la recherche en sciences sociales, $\mathrm{n}^{\circ} 151-152,2004$, pp. 36-48.

17. Le programme communautaire TACIS (Technical Assistance to the Commonwealth of Independant States-CEI) a été créé en décembre 1991. Instrument financier d'assistance technique, il a pour objectif d'encourager «l'établissement de conditions favorables à l'économie de marché et de renforcer la démocratie » dans les pays concernés. http:// www.ladocumentationfrancaise.fr/dossiers/europe-russie/programme-tacis.html. En 2000, le consultant référent pour la Mongolie est français. Nous le rencontrons lors d'un cocktail organisé pour le 14 juillet à l'ambassade française. Il raconte qu'il a passé quelques années en Russie avant de prendre le poste à Oulan-Bator. Il dit alors à propos des Mongols : « Ils sont incultes, ils n'ont pas de piano dans les appartements et il n'y a pas de fleur dans la steppe ».

18. Le Japon est le premier pays donateur en Asie pour des programmes d'aide au développement. Sa politique internationale de «soft power » vise la captation des matières premières à l'échelle du continent. Il est en concurrence avec la Chine, plus agressive sur le plan commercial. À ce sujet, voir Faure G., «La Banque asiatique de développement et l'intégration régionale en Asie ", Études internationales, vol. 38, n², 2007, pp. 229-249. Mais ses actions sont si « discrètes » que le Japon n'est pas identifié comme un « envahisseur » en Mongolie; il n'a été que très peu mentionné lors de nos entretiens et toujours en termes «neutres », factuels : " nous avons une subvention », "deux représentants sont venus nous rendre visite ».

19. Précisons qu'elles se sont constituées en " association ", c'est-à-dire légalement indépendamment du parti politique, et qu'elles sont donc ouvertes à une participation de femmes qui n'en seraient pas membres. Dans les faits, ce n'est pas le cas, et leurs activités « en dehors du parti » n'en demeurent pas moins identifiées comme affiliées à celui-ci. De plus, elles constituent à nouveau le vivier des femmes politiques que le Parti promeut, ce qui prouve, si besoin en était, le rapport tutélaire que les dirigeants entretiennent avec ces femmes. 
Dans un premier temps, ce partenariat international a semblé très porteur. Il est manifeste que si les femmes s'investissent avec tant d'ardeur dans ces associations, c'est aussi parce qu'elles ont perdu leurs postes de fonctionnaires (enseignantes, juristes, médecins, etc.) et que ces programmes de développement leur permettent de recycler leurs compétences administratives et de service public. Le nouvel espace public des associations devient un secteur d'emplois salariés, ce qui peut même mener au constat que « l'activité concrète sur les projets n'est souvent qu'une excuse pour se créer un emploi 20 ». Les associations de femmes vont développer de nouveaux objets de luttes dont elles prennent connaissance lors des rencontres internationales et par le biais des propositions de programmes internationaux, comme la défense juridique des femmes victimes de violences domestiques ou de licenciements abusifs, la lutte contre la prostitution des mineurs, la baisse des montants des allocations familiales et l'appauvrissement des femmes et de la population en général 21. Elles vont ainsi acquérir une certaine expertise, comme le montrent par exemple les curriculum vite des jeunes salariées du Centre contre les violences domestiques, qui présentent dès 1999 des diplômes de masters tout récemment obtenus en Europe et aux États-Unis en "gender and development studies ", «international human rights » et «NGO's management ».

\section{Constitution d'un capital symbolique}

Cet espace international constitue une seconde ressource : il s'agit de sa dimension symbolique, autrement dit l'image, le «prestige » (des États-Unis par exemple) que les associations de femmes vont essayer d'utiliser pour faire pression sur l'espace national et plus précisément sur les occupants de l'espace du pouvoir, c'est-à-dire les leaders étatiques et des partis politiques. À la lecture de la multitude de documents produits par les associations de femmes (plaquettes de présentation, rapports d'activités, lettres à différentes institutions, articles de presse, documents internes de travail, etc.), on remarque une première utilisation symbolique des pays étrangers : les militantes mongoles les citent en exemple de « réussite » en "égalité de genre ». La plaquette de présentation de la Coalition des femmes, généreusement distribuée lors de toute rencontre politique et publique, explique que : «L'État ne perd rien à faire entrer les femmes en politique. Les pays développés nous le prouvent, comme les pays scandinaves où le problème du logement est totalement résolu et où le système d'éducation est gratuit ». Mönhöö, ancienne députée parlementaire communiste et créatrice d'une petite association de femmes intitulée Mouvement Gal Golomt (Mouvement pour le foyer), déclare dans la presse un mois avant les élections : «Je pense que le nombre de femmes en politique

20. Ditchev I., « La nouvelle classe de médiateurs », Transitions, n42, 2001, p. 100.

21. Cette dernière, qui s’organise en particulier avec la «bénédiction » d'ONG religieuses et/ou républicaines américaines (l'USAid par exemple), se développe sur le mode caritatif et crée ainsi une catégorie qui n'existait pas du temps du communisme - celle des assistés, et plus spécifiquement des «femmes assistées »-, ce qui renforce une idéologie de genre patriarcale. 
est largement insuffisant. La moyenne internationale est de $10 \%$, en Scandinavie, elles sont $39 \%$. [...] La participation des femmes à la politique n’a jamais été néfaste et les problèmes sociaux sont résolus 22 ». Zanaa, activiste démocrate de la première heure et présidente du très dynamique Comité de veille $\mathrm{CEDEF}^{23}$, se positionne à l'opposé dans le paysage idéologique et politique. Elle s'exprime pourtant de la même façon dans la presse que sa collègue communiste :

«Les femmes ont un devoir naturel de reproduction, c'est un devoir social spécifique (qui doit être accepté en tant que tel). Elles doivent donc être traitées différemment des hommes. [...] Mais ces lois oublient les pères, qui s'occupent aussi pourtant de leurs enfants, ou devraient le faire. Il en est autrement au Danemark par exemple, où les parents peuvent discuter pour décider qui va prendre un congé spécifique pour s'occuper d'un jeune enfant. Grâce à ces lois progressistes, il y a une obligation de dialogue au sein des couples et on constate moins de violence domestique et sociale 24 ».

L’idéalisation de ces nouveaux partenaires qui se présentent eux-mêmes comme tels, permet ici la construction d'un « modèle à suivre ", modèle d'égalité et/ou modèle de développement économique entraînant l'égalité entre hommes et femmes. La France, par exemple, est connue par les Mongoles pour son mouvement féministe intellectuel des années 1970 et sa «liberté sexuelle $» .$.

En citant ces « modèles » étrangers, les militantes vont également inclure leurs actions dans celles du mouvement international de la lutte pour les droits humains en général, et pour les droits des femmes en particulier. Par exemple, dans une interview pour le quotidien Zuuny Medee, le 23 mai 2000 (en pleine période de campagne électorale), Zanaa explique que « [l]a Coalition [des associations pour le soutien aux femmes candidates] a été créée le 10 décembre 1999, le jour même de la célébration de la journée internationale des droits de l'humain, par les directrices et présidentes des associations de femmes qui s’étaient déjà mobilisées pour les élections de 1996 ». Le logo de la coalition présente la forme d'un drapeau et, sur la moitié gauche, 40 petits motifs en rang rappellent fort les étoiles du drapeau américain. Il s'agit en fait de petites fleurs, chacune représentant une association. Un dernier exemple de cette utilisation de l'international est celui de la Fondation pour les femmes rurales. Sa mission est officiellement de « [t]ravailler pour l'implantation des prévisions de la Déclaration de Genève sur les femmes rurales 25 ». Afin de faire valoir la

22. Le quotidien Önödöör, 23 juin 2000.

23. Convention internationale sur l'élimination de toutes les formes de discrimination à l'encontre des femmes, adoptée par l'Assemblée générale des Nations Unies en 1979.

24. Önödöör, 15 février 2001.

25. Plaquette de présentation des activités du Centre d'information et de recherche sur les femmes, Oulan-Bator, nov. 1998. 
qualité de leurs études et projets, les associations font toujours référence à un partenariat avec des consultants et organisations étrangères, ce qui correspond généralement à la réalité. Dans la plaquette de présentation de son association Genre et développement, la présidente Erdenčimeg explique qu'elle a assisté à une formation sur le « genre » mise en œuvre par une consultante ougandaise, qui elle-même avait fait ses études à Londres. Dans le quotidien Mongol Messenger ${ }^{26}$, la très importante Association des femmes libérales et intellectuelles (dénommée par son sigle mongol, LEOC, y compris dans les documents anglophones) annonce la création d'une librairie itinérante dans trois régions du pays avec l'aide d'un programme de développement allemand. Toutes les associations accueillent une à deux consultantes étrangères qui participent aux réunions inter-associatives dans la capitale, mais qui se déplacent aussi avec plaisir à la campagne, en visite plus ou moins touristique chez les militantes. Ces militantes, jusqu'au « fin fond de la steppe », ont ainsi connaissance de ces partenariats avec des étrangers et ont résolument le sentiment de s'inscrire dans un vaste réseau international spécialisé sur le genre, comme cette militante locale : «oui, [...] nous publions des articles en anglais. Il faut que les femmes travaillent ensemble 27 ». Par exemple, en 1998, la Fédération des femmes mongoles, grande association ayant repris les activités de l'ancien Comité des femmes du PPRM faisait état de 11 projets en cours, dont un seulement ne bénéficiait pas d'un partenaire international 28. Ou encore : «Concernant les rencontres internationales, le Centre d'information et de recherche sur les femmes a participé 1) au second Forum des femmes asiatiques ; 2) à la consultation régionale de l'Indochine et de la Mongolie sur la mise en œuvre et le suivi post-Pékin ; 3) à la conférence "Promouvoir les femmes dans le domaine de l'information” (Séoul, Corée du Sud 1996) ; 4) au premier forum du Programme pour les femmes pour un réseau d'OSI 29 (Budapest, Hongrie 1997); et 5) au quatrième Congrès international sur le sida en Asie et dans le Pacifique (Manille, Philippines 1997) ». Elles concluent

26. Daté du 17 janvier 2001.

27. Ojuunceceg, sous directrice branche locale de LEOC à Zuun Mod, dans la région Töv (centre du pays), rencontrée à son bureau le 17 janvier 1999.

28. Voir sa plaquette de présentation de 1999. Voici les 11 projets en question : «le "MON/93/WO-1", pour améliorer le niveau de vie des femmes mongoles, avec l'UNIFEM, en 1991-1994; le "MON/93/PO-5" pour augmenter leurs revenus avec le Fonds pour la population des Nations Unies et le BIT, en 1993-1994 ; le "DAP" avec l'ambassade australienne à Pékin, petit projet pour la formation au maraîchage et l'utilisation du cachemire; le “340/000/04", pour fournir une assistance technique aux femmes, avec l'organisation religieuse allemande "Mesereor", en 1994-1995; une formation en prophylaxie sanitaire auprès des prostituées sur la prévention SIDA, avec l'OMS et le Ministère de la Santé en 1995; un projet de réduction de la centralisation des associations de femmes et renforcer leurs capacités locales, avec TACIS (Europe); un accompagnement aux petits projets visant à augmenter les revenus des femmes rurales, avec l'ambassade australienne à Pékin ; une formation à la gestion d'entreprise et un projet de microcrédits avec "Italian Society for Progress" de TACIS, en 1996-1997 ; un programme de protection de la santé avec l'ambassade hollandaise à Pékin ; un programme d'éducation parentale, avec l'UNICEF; un projet de lutte contre la désertification du Gobi, avec le gouvernement mongol ; un projet de microcrédit avec le PNUD en 1998 » (FFM, non daté, consulté en 2000).

29. OSI : organisation de solidarité internationale. 
ainsi leur plaquette : «Le Centre souhaite la coopération de toute organisation travaillant aux mêmes objectifs ». Citons également le mouvement Gal Golomt, qui compte neuf bénévoles : "Nous collaborons avec l'association mongolo-japonaise Solongo (Arc-en-ciel) et sommes désireuses de promouvoir et étendre plus loin les relations externes ». Les militantes au sein des partis politiques aussi « s'internationalisent » : les trois femmes du bureau du parti des Verts travaillent à un projet avec une association allemande de protection de l'environnement, auraient des relations avec les partis des Verts de Taiwan, de Corée du Sud, de Suède, et reçoivent des brochures anglophones transmises par les Suédois ${ }^{30}$. Le 10 avril 2000, lors d'une réunion de travail de l'association des femmes du parti social-démocrate ${ }^{31}$, une intervenante rappelle qu'elle a assisté à une conférence internationale socialiste à Paris en 1999. Elle égrène les thèmes propres à la lutte des femmes dans le monde qui y avaient été abordés et qui concernaient également la Mongolie : pauvreté due aux changements macro-économiques, viols, maitrise de la fécondité et santé reproductive, femmes rurales, indépendance économique, participation dans les instances de prises de décisions, etc. ${ }^{32}$ Même Ojuun, dirigeante du très libéral Parti du courage civique et déjà forte du capital symbolique hérité de son frère Zorig assassiné et promu héros de la nation ${ }^{33}$, met l'accent sur ses liens à l'international. Dans ses publications, on peut la voir en photo en Malaisie, en Russie, en réunion avec l'ambassadeur américain en Mongolie, en rencontre culturelle avec des Japonais et des Coréens, etc. Ces femmes de l'élite intellectuelle et politique, qui avaient déjà fait l'expérience de relations internationales lors de leurs études supérieures - même si elles étaient restées à l'intérieur du bloc soviétique - ne font que prolonger aujourd'hui cette pratique avec de nouveaux partenaires.

La démarche n'est pas à sens unique, puisque les organisations internationales leur décernent en retour des « honneurs ", attestant et renforçant leur inclusion symbolique au sein de cet espace international. Dans leur plaquette de présentation éditée en 1999, un encadré nous apprend par exemple que « [1]e Mouvement des femmes pour le progrès social [MFPS] a gagné le EUUS Democracy and Civil Society Awards. Le concours a été initié au Sommet États-Unis/Union européenne de mai 1998 à l'occasion du 50e anniversaire du plan Marshall et du 40e anniversaire du Traité de Rome. Cinquante gagnants ont été désignés sur les 140 candidats par les ambassades américaines et européennes de 28 pays. Le MFPS a été sélectionné pour sa contribution à une bonne gouvernance, à l'éducation citoyenne et à la défense des droits des femmes dans la capitale et dans les régions ».

30. Rencontrés à leur bureau de la capitale le 5 mai 2000.

31. Le Mouvement des femmes sociales-démocrates reprend ainsi la pratique de l'organe féminin au sein du parti politique, mais il s'agit ici de l'un des deux partis démocrates majeurs.

32. Badamhorol, le 10 avril 2000 à Oulan-Bator.

33. En 1999, le gouvernement l'honore du titre de « Meilleur homme politique du siècle », une statue est érigée à sa mémoire le 28 avril, jour de son anniversaire (il aurait eu 37 ans) et 20000 timbres sont imprimés en Hongrie à son effigie. 
Ces militantes se sont ainsi constitué un véritable capital symbolique, avec lequel elles tentent de faire pression sur leur gouvernement en vue d'obtenir des résultats tangibles. Comme par exemple le Centre contre les violences domestiques (Center Against Violences, CAV, qui conserve son sigle anglais quand on en parle en mongol), qui déclare qu' «il n'y a pas de programme gouvernemental spécifique de réduction des violences domestiques et que le soutien gouvernemental aux associations portant cette question demeure très insuffisant ${ }^{34}$ ». L'association qui va le plus utiliser cette inscription internationale est sans aucun doute le Comité CEDEF, véritable « observatoire du genre » constitué en association et qui se donne pour mission de se concentrer sur l'application de la Convention signée par la Mongolie dès 1981. En 1999 et 2000, son rapport d'activité indique qu'il a consacré les deux tiers de son budget de 50000 US\$ aux « relations internationales », c'est-à-dire faire venir des consultant(e)s mais aussi financer la participation des militantes mongoles à des rencontres internationales. À la recherche des stratégies militantes les plus efficaces, il va tenter de mobiliser en doublon : d'une part, l'espace national rural et urbain, en sensibilisant les nouveaux citoyens par l'organisation de nombreuses conférences locales, mais aussi et d'autre part, l'espace politique international. En effet, l'arme principale de sa créatrice en Mongolie, Zanaa, consistera à interpeller son gouvernement en rappelant son engagement non pas auprès des femmes du pays, les citoyennes, mais auprès de la communauté internationale. Elle déclare par exemple clairement dans le quotidien Önödöör que « [l] gouvernement doit tout mettre en œuvre pour réaliser l'égalité entre les hommes et les femmes, il en a fait la promesse 35 ». Dans son rapport annuel de 2000, le Comité CEDEF fait un compte-rendu efficace de ses activités, en sept axes de travail dont la «Coopération nationale et internationale ». Les rédactrices listent les nombreux rapports rédigés dans le cadre du suivi international prévu par la CEDEF, mais aussi de la préparation de la Conférence de Pékin +5 et de l'UNGASS ${ }^{36}$. Sur la seule année 2000, elles comptabilisent douze rencontres internationales, dont quatre à l'étranger : Hong Kong, New York, Taipei et Kuala Lumpur. Et lorsqu'en 2010, on tape son nom sur Google, Zanaa apparaît dans pas moins d'une dizaine d'organisations internationales parmi lesquelles Women's E Gender Studies Association of Countries in Transition ${ }^{37}$, Global Fund for Women ou encore l'Asia Pacific Women Law and Development ${ }^{38}$. Elle sera celle qui, croisant l'espace politique international avec l'espace politique national, utilisera le plus les références symboliques

34. Association des femmes juristes et CAV, 2003, plaquette de diffusion: Ways for Prevention of Domestic Violence. Handbook.

35. Daté du 15 février 2001.

36. Session spéciale de l'Assemblée générale des Nations Unies sur le SIDA, 25-27 juin 2001, New York.

37. Créée en 1998, à la suite d'un rassemblement à Belgrade en décembre 1998, sur impulsion de l'association Center for Women's Studies, www.zenskestudie.edu.yu/wgsact/index.html. Le Comité CEDEF en fait partie, comme 29 autres pays. 
dont sont porteurs les pays occidentaux en Mongolie afin de faire pression sur son gouvernement.

Cette double stratégie des associations de femmes, d'une constitution en mouvement associatif en parallèle d'une inscription dans un espace international, témoigne de quelques résultats indéniables, confirmant « l'effet boomerang ». Dans un premier temps, le gouvernement est mis devant le fait accompli et tente de s'en féliciter : "Plus de 170 lois ont été adoptées depuis l'adoption de la Constitution en 1992 et beaucoup d'entre elles comprennent des clauses interdisant la discrimination de genre 39 ». Il lance quelques programmes ambitieux, comme le programme national «Améliorer l'État pour les Femmes [...] approuvé [...] en 1996 et le Rapport sur le développement humain préparé par le PNUD en 199740 ». Cinq années plus tard, " [1]e Programme national de promotion de l'égalité des sexes a été adopté en 2002, en application de la résolution $\mathrm{n}^{\circ} 274$ du gouvernement conformément aux tendances modernes en matière de développement et à la nécessité stratégique de la promotion de la femme. [...] Le programme est en cinq parties ${ }^{41}$ incluant les 12 domaines critiques du Programme d'action de Pékin et son exécution est prévue en cinq étapes d'ici à 201542 ». Cependant, aucun résultat n'est signalé dans le bilan effectué en 2004.

38. «APWLD is an independent, non-government, non-profit organization. It is committed to enabling women to use law as an instrument of social change for equality, justice and development. It has a consultative status at the Economic and Social Council of the United Nations (ECOSOC). APWLD developed from dialogues during 1985-1988 among Asia Pacific women lawyers, social scientists and activists, beginning at the 1985 Third World Women Forum on Women, Law and Development held in Nairobi, Kenya " (www.apwld.org/aboutus.htm). On la croise sur le site du US Department of State, dans la retranscription d'une vidéo conférence qui a eu lieu le 14 mai 2002, avec Lorne Craner, Assistant Secretary of State for the Bureau of Democracy, Human Rights, and Labor, "who is here to discuss U.S. human rights and democratic policy and the roles they play in the U.S.-Mongolian relationship ». On trouve encore CIVICUS, the Center for Health and Gender Equity (ONG américaine avec donations privées de fondations), Global Media Monitoring Project (constitué d'associations de partout dans le monde), Les Pénélopes y font référence à propos de la participation de Zanaa au Forum de AWID à Mexico en 2002...

39. «Pour citer quelques exemples : Articles 14 et 16 de la Constitution, Paragraphe 2 de l'Article 1 de la loi électorale, Article 19 de la loi sur les tribunaux, Art. 56 du code civil, Art. 15 du code criminel, Art. 3 de la loi sur les relations en l'État et des Églises, Paragraphe 1 de l'Art. 4 de la loi sur les voyages à l'étranger, la propriété privée et l'émigration des citoyens mongols ». Non daté-1998, Version provisoire des Examens des rapports présentés par les États parties conformément à l'article 18 de la CEDEF pour 1999, (ministère de la Santé et de la Protection sociale, version provisoire donnée le 17 décembre 1998 par G. Altanceceg), OulanBator, anglais.

40. Association des femmes juristes, 1998, Plaquette de présentation, Oulan-Bator, anglais et mongol.

41. Les titres en sont : «l'égalité des sexes dans les relations familiales, [...] dans les relations économiques, $[\ldots]$ en matière de développement rural, $[\ldots]$ au niveau de la prise de décisions, et la participation de la société civile».

42. CEDAW/C/MNG/7, Comité CEDEF, 2007, Nations Unies, Examen des rapports présentés par les États parties conformément à l'article 18 de la CEDEF. Cinquième, sixième et septième rapports périodiques des États parties. Mongolie, français, p. 13 (http://daccessdds.un.org/doc/UNDOC/GEN/N07/315/81/PDF/N0731581.pdf? Open Element). 
Le gouvernement a également été sommé de créer des institutions adéquates.

«Le Conseil national sur l'égalité des sexes créé en 2001 et dirigé par le vice-président du conseil des ministres n’était pas un mécanisme approprié pour intégrer une perspective sexospécifique dans les différentes politiques [...]. Par conséquent, le gouvernement a créé en 2005 la Commission nationale sur l'égalité des sexes dirigée par le Premier ministre, $[\ldots]$ organe consultatif national [...]. Elle a pour fonction d'élaborer et d'appliquer la politique de l'État dans ce domaine, de soutenir les initiatives [de tous les acteurs], et de coopérer avec les organisations internationales et les donateurs, et de coordonner leurs activités ${ }^{43}$. »

Dans un autre registre, la responsable des relations publiques de l'Association des femmes juristes signale que le Premier ministre Enhbajar a prononcé un discours à l'occasion du 8 mars 2001, dans lequel il a manifesté la volonté de l'État de lutter contre la violence domestique. Elle y voit la reconnaissance étatique de ce problème national. Mais elle précise que l'association note surtout des difficultés rencontrées par les femmes auprès de la police, dont les agents ne reconnaissent pas le statut de victime et qui les découragent dans leur démarche pour porter plainte ${ }^{44}$. Cette réalité réduit à néant les opérations de communication gouvernementales.

Les dirigeants politiques sont obligés de reconnaître publiquement le travail de ces associations de femmes et de leurs dirigeantes. Le 25 novembre 2004, le président Bagabendi en personne décore Zanaa de l'Ordre de l'étoile rouge, en reconnaissance de sa contribution au développement de la démocratie, de la société civile et des organisations non gouvernementales en Mongolie. Tungalag ${ }^{45}$, présidente du Mouvement des femmes socialistesdémocrates, a reçu un peu plus de soutien de la part des dirigeants de son parti en 2000 qu'en 1996. Elle avance trois explications à cette évolution : 1) l'instrumentalisation des femmes car ils se sentent en difficulté à la suite d'une instabilité gouvernementale grave en 1998-2000 alors qu'ils sont au pouvoir ; 2) les effets du travail de la Coalition des femmes; et enfin 3 ) la pression de l'Internationale socialiste dont ils sont membres et dont ils sont obligés de suivre les directives en termes de parité. Mais cette pression n'aura finalement aucun effet, puisque le Parti Social-Démocrate (PSD) ne soutiendra que trois femmes sur 76 candidats.

43. Ibid., p. 13

44. Rencontrée à Oulan-Bator, le18 avril 2001.

45. Rencontrée à Oulan-Bator, le 6 avril 2000. 


\section{Contre-effet boomerang}

En fait, à l'image du PSD, le résultat de cette stratégie de « pression » sur l'ensemble de l'espace politique et du pouvoir sera bien plutôt un échec. En témoigne cet échange entre Zanaa (et le Comité CEDEF) et le gouvernement. En 2001, elle rédige un rapport alternatif (Shadow Report) sur le respect de la CEDEF en Mongolie 46. "Ce travail fut accompli seulement par les bénévoles du Comité et aucun fonds n’a été utilisé 47 ». Elle poursuit : " Des représentantes du Comité CEDEF ont assisté à la 24e assemblée CEDEF qui a eu lieu en janvier 2001 à New York [...] Leur participation a été financée par IWRAW-AP 48 ». Elles concluent ainsi leur rapport d'activité 2000/2001 : «La rédaction du rapport alternatif a eu pour résultat que les Nations Unies et le Comité CEDEF ont adressé des commentaires spécifiques au gouvernement de la Mongolie $49 »$. Elles y proposent des amendements au code du travail, au code de la famille, à la loi sur la protection sociale. Elles produisent un résumé de ce rapport, puis un commentaire, qu'elles distribuent « aux candidats à l'élection présidentielle de 2001, aux ministères et aux associations de femmes 50 ». Cependant, elles écrivent également et pour la première fois dans un document publié : «Nous avons reçu une réponse officielle de la part des ministères de la Justice, de la Protection sociale et du Travail. Son contenu est une véritable humiliation pour le Comité CEDEF et pour ses activités 51 ». Le ministre adjoint signe en effet une lettre dont le ton et les propos sont particulièrement méprisants, c'est-à-dire, en termes analytiques, qui déconstruit le capital symbolique qu'elles avaient tenté de constituer. Les six recommandations qui leur ont été adressées sont reprises et ridiculisées. Concernant par exemple la première, portant sur la loi sur les élections parlementaires, la réponse indique sans détour que " [l]e défi majeur de chaque parti politique est de gagner les élections [...]. Il est évident que [dans ce but] un parti doit désigner les candidats qui détiennent les capacités intellectuelles et la popularité les plus hautes».

Le Comité CEDEF avait également critiqué l'attitude paternaliste de l'État vis-à-vis des femmes en n'attribuant qu'un congé maternel, renforçant ainsi « l'idée que seules les femmes doivent s'occuper des enfants ». Il répond alors : «Il est déplaisant de tirer des conclusions extrêmes et irréalistes et de penser que les hommes pourraient "faire le travail” d'être enceinte et de nour-

46. Rapport alternatif : traduction de Shadow Report, rapport rédigé uniquement par la société civile et qui se distingue donc d'un rapport co-écrit avec des acteurs gouvernementaux.

47. Zanaa J. et Comite CEDEF, Shadow Report to the Mongolian Government's 3rd Report to the CEDAW Committee in the United Nations, Oulan-Bator, 2001, p. 3.

48. International Women's Rights Action Watch-Asia Pacific, étroitement lié à CEDEF, financé par des entités gouvernementales (UNIFEM par exemple) mais aussi des fondations privées telle que la Fondation Ford (www.iwraw-ap.org/aboutus/donors.htm)

49. Ibid., p. 7.

50. Ibid., p. 2.

51. CEDAW Watch Committee, Annual Report of the Activities, 2000 and 1st quarter of 2001, Oulan-Bator, 2001, p. 4. 
rir un bébé, alors que la nature de leur structure biologique n’y est pas prédestinée. En outre, ces clauses constituent sans aucun doute une véritable protection pour les femmes. Ces remarques réduisent la portée du rapport et constituent un obstacle à sa publication à un niveau international, mais elles montrent aussi votre incompétence et votre faible intelligence ». En post scriptum, la réponse gouvernementale indique que « [c]e rapport alternatif contient de nombreuses erreurs d'écriture et d'expression, et vous devez le re-écrire en portant attention au fait qu'il ne correspond pas aux directives requises [...]. En réécrivant ce rapport, vous devez exclure le paragraphe 3 des recommandations adressées au gouvernement ». Cette dernière adresse est indéniablement d'un immense mépris pour le travail des associations de femmes, qui marque leur échec. On est ici, semble-t-il, face à un problème de légitimité de ce mouvement associatif et de ses militantes, pour qui toute la difficulté réside dans leur positionnement au sein de cette nouvelle démocratie. Lorsqu'elles travaillent en collaboration avec l'État, elles rencontrent un problème de censure éventuelle ou de complaisance, et se font parfois même accuser de compromission, surtout si elles en obtiennent des financements. Mais lorsqu'elles se mettent en position de rupture avec l'État, celui-ci leur renvoie un argument de non-représentativité.

Un des intérêts de la production d'un Shadow Report, c'est justement de pouvoir se démarquer de la politique nationale et d'en dénoncer certaines pratiques ${ }^{52}$. Autrement dit, le rapport critique les représentations et les actions des acteurs dominants. Or, ce rapport alternatif est rédigé en 2000 par des femmes qui étaient en 1990-1992 - pour la majorité d'entre elles - au coude à coude avec les jeunes révolutionnaires démocrates à qui elles s'adressent maintenant dans le rapport puisqu'ils sont au pouvoir. Anciens partenaires démocratiques, ils sont devenus aujourd'hui des adversaires sexistes. Ceci est assez révélateur de la problématique stratégique dans laquelle le mouvement associatif des femmes se trouve embourbé. "Le problème est que, si les associations ont donné naissance à l'État social, l'État ne les a reconnues que dans un rapport tutélaire 53 ». Et c'est un obstacle à la légitimité de leurs revendications. Les représentants légitimes de l'État ont donc réussi à contrer l'effet boomerang qu'avaient tenté de mettre en place les associations de femmes.

\section{Nation contre genre}

Il manque un dernier élément à l'analyse de cet échec : il concerne l'outil qui a permis ce « contre-effet boomerang ». Car pour anéantir un mouvement associatif, surtout s'il est fort d'un capital symbolique international, il ne suffit pas d'en avoir la volonté. Il faut détenir une arme plus puissante encore que

52. Nous tenons ici à remercier Caroline Ressot, docteure en droit international, qui nous a éclairée sur cette question.

53. Laville J.-L., Caillé A., Chanial P., Association, démocratie et société civile, Paris, La Découverte/MAUSS/CRIDA, 2001, p. 12. 
le «boomerang ». Et cette arme, c'est celle de la «tradition». Elle nous a été présentée par le premier secrétaire du PPRM, Enkhbajar ${ }^{54}$, qui a accepté une rencontre sur le thème que nous lui avions annoncé : «les femmes en politique ». Nous avions constaté avec lui le faible nombre de femmes candidates pour son parti, à trois semaines des élections, soit 8 pour 76 . Pour explication, il ne s'est pas perdu en considérations démagogiques (et nous lui en sommes reconnaissante). Son unique argument a été simple : « une candidature féminine est une prise de risques, dans un pays asiatique où les traditions sont patriarcales ». Le PPRM n'est pas le seul à craindre ce « risque », puisque chez les démocrates aussi, "cette crainte est d'autant plus grande pour les sièges à la campagne qui est considérée comme plus attachée encore à la tradition 55 ». Cette remarque est d'autant plus surprenante que la Mongolie est dans une double relation de crainte et de mépris à l'égard de la Chine - crainte de l'invasion démographique et économique que les Mongols ont déjà connue du $\mathrm{XVII}^{\mathrm{e}}$ au XXe siècle et qui a laissé la population exsangue - qui se transforme en un « racisme ordinaire ». À la fin des années 1990, on assiste même à l'émergence d'une dizaine de petits partis politiques dont le seul discours portait sur le refus de la "présence chinoise » et la défense de la nation. Lors de notre premier séjour dans le pays (1998-2000), les témoignages sont nombreux de distinction nationale (et raciste) en opposition à la Chine en particulier, référence faite aux rapports entre hommes et femmes et à la «culture nomade »: les Chinois sont agriculteurs, commerçants et sédentaires, leurs femmes sont opprimées par la pratique des pieds bandés, tandis que le «Peuple mongol» est éleveur et nomade, et ses femmes sont intelligentes et libres ${ }^{56}$.

Lors de la rencontre avec le futur président de la République, cette référence soudaine à une "culture asiatique » qui efface la distinction avec la Chine, est donc un élément nouveau dans le «processus de transition». Il révèle le refus de remettre en question les rapports sociaux de sexe patriarcaux, y compris en temps de transition politique (ici démocratique) ${ }^{57}$. En outre, l'identité nationale, ici considérée comme "asiatique ", serait donc fondée sur la domination masculine (face à la sociologue française). C'est donc qu'un ennemi potentiel « hors Asie » a fait son apparition. Sachant que la Russie n'est plus qu'un « grand frère » dont on connaît les propres difficultés - par

54. Rencontré le 7 juin 2000 à Oulan-Bator. Il sera ensuite Premier ministre de 2000 à 2004 et président de la République de juillet 2005 à 2009.

55. Entretien avec Bajarceceg, salariée de l'association LEOC, le 7 avril 2000 à Oulan-Bator.

56. Pour une analyse de l'idéologie de genre en Mongolie, voir les travaux de Roberte Hamayon, «Le pouvoir des hommes passe par la "langue des femmes". Variations mongoles sur le duo de la légitimité et de l'aptitude ", L'Homme, vol.19, n³-4, 1979, pp. 109-139; et Hamayon R., Bassanov N., "De la difficulté d'être une belle-fille ", Cabiers d'Études Mongoles et Sibériennes, vol.4, 1973, pp. 7-73.

57. Ainsi, on assiste à une pérennité de la domination masculine traversant les bouleversements politiques, économiques et sociaux, telle que l'ont montré, pour l'Europe par exemple, les travaux des historiennes telles que Michelle Perrot, (« Les femmes et la citoyenneté en France. Histoire d'une exclusion », in Le Bras-Chopard A. et Mossuz-Lavau J., Les femmes et la politique, Paris/Montréal, L’Harmattan, 1997, pp. 23-39). 
exemple, avec la pauvreté des régions frontalières comme la Bouriatie et Touva, dont les jeunes hommes viennent voler le bétail au risque de se faire tuer par la police mongole -, il faut le chercher ailleurs : il s'agit des nouveaux partenaires internationaux, les États-Unis en tête de file. Au terme de dix années de transition, la population est obsédée par une crise économique qui ne se résorbe pas malgré les effets d'annonces. Les réformes économiques continuent d'accentuer le creusement des écarts de richesses. Et l'État, soumis à des plans de réajustements structurels, continue de réduire ses dépenses publiques, ce qui maintient les personnes dépendantes des aides publiques dans une grande pauvreté. Le formidable espoir qui avait porté la transition se volatilise et les fameux pays « modèles » perdent peu à peu leur capital symbolique. C'est à la faveur de ce contexte économique que réapparaissent la rhétorique nationaliste et la réactivation de la «tradition nomade ", refuge identitaire et idéologique autant qu'économique ${ }^{58}$. Cette «tradition » ne remet pas encore en cause, en Mongolie, la notion d'idéal démocratique. Mais elle cultive la domination masculine, comme partout dans le monde ${ }^{59}$.

Profitant de ce contexte pour déstabiliser les démocrates, le PPRM réactualise un discours sur l'idée d'un développement autonome, sans ingérence étrangère susceptible de provoquer un changement trop brusque. Avec cet argumentaire, l'ancien parti communiste remporte 68 sièges sur 74 au parlement en 2000 , véritable « raz de marée rouge » inattendu; seules huit femmes seront élues. On le comprend, ceci ne va pas servir la stratégie adoptée par le mouvement associatif des femmes. Les autorités locales « associent 》 les revendications égalitaires des femmes avec cette ingérence étrangère et considèrent leurs activités comme "brevetées par l'Occident 60 ». C'est dans ce contexte que l'identité mongole peut alors oublier ses vieilles dissensions avec la Chine et promouvoir une «tradition » asiatique, d'autant plus nouvelle qu'elle associe nomadisme et communisme, alors que le second s'était justement construit contre le premier au début du siècle, en particulier contre la figure guerrière de Gengis Khan remise à l'honneur à la faveur de la transition libérale ${ }^{61}$. Cette «tradition nationale " se réactualise ainsi au fil des évolutions politiques. C'est bien contre elle que Zanaa bute dans son combat, même si elle tente de l'attaquer de plein fouet.

58. Au grand étonnement des observateurs, on a assisté à un retour au nomadisme, c'est-à-dire à l'élevage qui s'est bien développé à la faveur de la libéralisation (voir les travaux de David Sneath, "Land use, the environment and development in post-socialist Mongolia," Oxford Development Studies, vol.31, n4, 2003, pp. 441-459). Malgré des pertes annuelles dues au froid, habituelles pour le pays, le cheptel n'a jamais été aussi important à l'échelle nationale, atteignant 39 millions de têtes de bétail pour une population humaine d'à peine trois millions de personnes. On craint désormais une dégradation écologique des pâturages.

59. Voir à ce sujet le travail fondamental de Yuval-Davis N., Gender and Nation, Londres, Sage Publications, 1997, p. 120-121, et plus précisément pour l'Asie centrale : Hours B., Sélim M., Anthropologie politique de la globalisation, Paris, L'Harmattan, 2010, p. 127.

60. Poujol C., «L'Asie Centrale, bilan : quinze années de discours et de pratiques sur l'intégration dans un espace désintégré », Revue internationale et stratégique, $\mathrm{n}^{\circ} 64,2006$, p. 73, et « Perception et traitement de l'aide internationale en Asie Centrale depuis 1991 : trajectoire d'une suspicion », Hérodote, n¹29, 2008, pp. 21-35. 
«Puisque la Mongolie a ratifié la CEDEF, nous ne pouvons pas échapper à ces responsabilités [de luttes contre les inégalités] en arguant de la tradition. Un homme devient un ennemi des femmes quand il viole les droits humains et ce, par n'importe quelle sorte de violence, légère ou grave. C'est un crime ${ }^{62}$. »

Le rapport alternatif évoque par exemple l'éventuel assouplissement de la règle patrilinéaire. "La clause 24.3 de la nouvelle loi sur la famille (1999) déclare qu'un enfant doit porter le nom de son père. Ceci viole l'article 16 de la Convention, qui déclare que les hommes et les femmes ont les mêmes droits et devoirs envers leurs enfants. Il est ainsi prouvé que l'État ne prend pas ses responsabilités [dans l'application de cet article de la Convention] ». Ce à quoi le ministre adjoint rétorque : "Il est important pour les enfants de respecter les pères et pour ces derniers d'assumer leurs responsabilités familiales, ce qui devrait faire baisser le taux de divorces. Les enfants qui portent le nom de leur mère identifient leur illégitimité, ce qui provoque de la souffrance en lien avec leur origine. Ceci est très profondément lié aux traditions nomades mongoles et à une culture ancienne. En particulier, être capable d'identifier son propre passé [...] Un autre aspect peut être celui de la politique ancienne de sécurité de la patrie ». Tout nationalisme vise à contrôler la population et donc la cellule familiale. Nira Yuval-Davis explique ainsi que la nation a souvent été conçue comme une extension de la parenté, car « ce sont les femmes et pas seulement la bureaucratie ni l'intelligentsia qui reproduisent les nations biologiquement, culturellement et symboliquement 63 ». De fait, « [1]e genre et la nation ne peuvent être analysés l'un sans l'autre, ils sont informés et construits l'un par l'autre 64 ». Jules Falquet rappelle que " [d]e son côté, Nicole-Claude Mathieu a [proposé] le concept de "l'arraisonnement des femmes" 65 ». Les études sur l'Asie centrale montrent comment un racisme déclaré a un impact sur les stratégies matrimoniales en construisant des interdictions de mariage entre partenaires de groupes identitaires différents 66. En Mongolie, nous n'avons pas observé de telles tensions. Pour ainsi dire, la question du nationalisme n’apparaissait pas non plus comme essentielle sur la scène politique nationale, du moins au début des années 1990, puisque la population était très enthousiaste à l'idée de l'ouverture à l'international. C'est bien l'approche par

61. Voir à ce sujet Françoise Aubin, «Renouveau gengiskhanide et nationalisme dans la Mongolie postcommuniste ", Cahiers d'études sur la méditerranée orientale et le monde turco-iranien, $\mathrm{n}^{\circ} 16,1993$, pp. 137-203, et Anna Jarry-Omarova, « Genre, nation et représentations en Mongolie », Raisons Politiques, n²4, 2006, pp. 159-175.

62. Önödöör, 15 février 2001.

63. Op. cit., p. 2.

64. Ibid., p. 21.

65. « À qui appartiennent les femmes ? L'“arraisonnement des femmes” par les mouvements sociaux mixtes et leur possible autonomisation : une analyse féministe », communication au colloque «Classe, ethnicité, genre... : les mobilisations au piège de la fragmentation identitaire ? ", 8-9 mars 2007, CRAPE, Rennes.

66. Voir à ce sujet Bazin L., « Mariage et identité nationale en Ouzbékistan. La figure de la bellefille, kelin ", La Revue, n³, 2009 (http://www.lrdb.fr/articles.php?lng=fr\&pg=1148), et Pétric B., Pouvoir, don et réseaux en Ouzbékistan post-soviétique, Paris, PUF, 2002, p. 142. 
le genre qui a révélé une certaine fragilité voir, même, une inquiétude nationale.

Si les observateurs américains pensaient alors que « [l]es nouvelles relations avec les États-Unis ont donné à la Mongolie de nouvelles options et de plus grandes chances d'établir son indépendance 67 ", ce n'est pas ce que pensent beaucoup de Mongol(e)s interrogé(e)s en l'an 2000, qui y perçoivent plutôt un danger. Comme en témoigne franchement Gantömör, religieuse bouddhiste rencontrée le 21 juillet 2000 dans le bureau du monastère de femmes qu'elle a construit et qu'elle dirige (chose sans précédent) : «avec l'influence étrangère, les femmes réclament l'égalité avec les hommes, elles fument et boivent comme les hommes. Ce n'est pas bien ». Badamdaš, jeune politiste rencontré le 30 mars 2000, explique qu'il a bien compris que la défense de « l'égalité de genre » s'appuie sur la notion des droits humains, avec l'idée que les femmes sont porteuses de progrès. Mais il réfléchit ensuite à haute voix : «Les principes de droits humains sont en contradiction avec la tradition... Je n'arrive pas à savoir si c'est positif ou négatif, mais c'est un constat. Par exemple, les principes des droits de l'enfant sont en contradiction avec ceux de respect des aînés que nous avons dans notre société. Nous sommes en train d'évoluer par rapport à ça, mais donc, nous perdons un peu notre culture ».

\section{En guise de conclusion}

En Mongolie, les ONG internationales n'ont pas été interdites, comme ce fut le cas en Asie centrale et en Russie dans les années 2004-2006 68. Mais le rejet de leur influence s'est porté sur le mouvement associatif des femmes et, à travers lui, sur l'ensemble de ses revendications d'égalité dans l'accès à l'espace du pouvoir. Les associations de femmes, celles de «type démocrate » en particulier, sont donc prises dans une contradiction qui les stérilise. Obligées de se tourner vers les organisations internationales du fait de leur capital tant financier que symbolique, elles diffusent la notion de genre, d'égalité, bref, d'idéal démocratique. Elles apostrophent leur gouvernement, mais se voient souvent qualifiées en retour de menteuses, de « mauvaises mongoles ». Or il n'y a pas meilleur argument de décrédibilisation que celui de «traîtrise à la patrie ». Les liens fondamentaux entre genre et nation ont ainsi permis la construction d'un « contre-effet boomerang "d'une efficacité redoutable puisqu'il a annihilé l'ensemble des stratégies du mouvement associatif des femmes.

Dans le même temps, la ressource financière, ou le «marché de projets » comme le nomme Ditchev ${ }^{69}$ a également commencé à se tarir. Burmaa, qui avait été à l'initiative d'une vaste éducation citoyenne au système électoral, à la

67. Jarrett K., “Mongolia in 1987. Out from the cold?”, Asian Survey, vol.28, n¹, 1988, p. 85.

68. Voir Bazin L., op. cit., 2009 ; Poujol C., op. cit., 2008 ; Hours B. et Selim M., op. cit., 2010.

69. Ditchev I., op. cit., 2001. 
publicisation des débats parlementaires en y faisant voter une loi autorisant la présence des journalistes, etc., déclare le 15 avril 2000 que son association, le MFPS, après avoir été financée très correctement, " est aujourd'hui en difficulté : nous n'obtenons plus de financements. Nous ne pouvons donc plus réaliser nos projets ». Elle aurait besoin de 10 millions de tugrik au minimum (soit 10000 US\$).

«L'Asia Foundation a financé 16 éditions de la revue bi-hebdomadaire Confiance et effort, qui avait pour vocation d'informer les citoyens des activités parlementaires et gouvernementales. Elle a acquis une vraie popularité parmi les législateurs et les électeurs. Nous ne pouvons plus l'éditer... ${ }^{70}$ ».

À l'échelle nationale, les mesures de réduction des inégalités sur le marché du travail restent inexistantes, mettant à mal l'engagement pris lors de la signature de la CEDEF et décevant bon nombre de militantes. Le mouvement associatif des femmes, à la suite de ces premières années d'intense activité, mais aussi face à cette multitude de difficultés, d'espoirs puis de déconvenues, est épuisé. Des conflits entre dirigeantes éclatent au grand jour, un scandale de détournement de fonds secoue l'ensemble du réseau, quand les femmes passaient pour être moins corrompues que les hommes. La ressource se fait rare, ce qui attise la concurrence.

Le problème spécifique au pays, et ce qui le distingue peut-être d'autres pays du Sud bénéficiaires des subventions internationales de développement, c'est que ces associations étaient les seules à composer l'espace public. En mettant à mal les associations de femmes, c'est en fait la déstabilisation de l'ensemble de cette société civile de la première décennie démocratique que le «contre-effet boomerang » a entraîné. Et ces femmes qui avaient pour unique objectif leur participation à la construction de la nation - mais une participation " par le haut ", au sein de l'espace politique et du pouvoir - sont rappelées à un ordre social " éternel et universel », y compris par leurs camarades démocrates, comme le déclare, bon enfant, Batbajar, ministre (démocrate) de la Santé et de la Protection sociale, qu'elles invitent pour le troisième Forum des femmes est-asiatiques en 1998. Il conclut sa longue intervention - donnant de nombreuses statistiques dans la pure tradition soviétique - par : «Je vous souhaite à toutes - merveilleuses mères et femmes, qui avez soigné et nourri l'humanité et qui lui avez permis de grandir, qui rendez le monde doux et tendre avec vos berceuses et permettez à l'humanité de vivre en paix et dans l'amour - je vous souhaite le meilleur ! 71 ». Il est ici clairement rappelé à toutes les militantes et femmes politiques présentes, dans un discours biologi-

70. MFPS, non daté-1999, p. 4.

71. LEOC, 1998, Report Brochure. The Third East Asian Women's Forum, Oulan-Bator, anglais et mongol, p. 6. 
sant, leur assignation à l'espace domestique et à la maternité. Le mouvement associatif des femmes paraît ne jamais avoir existé...

Ces intellectuelles continuent cependant de travailler, mais de manière moins visibles et surtout moins officiellement ciblées sur la question des droits des femmes. Le comité CEDEF, par exemple, a changé de nom, pour un Centre pour l'alliance des citoyens, dont le site internet 72 consulté le 7 juillet 2011 est « en travaux ", c'est-à-dire vide de tout contenu. En fouillant un peu plus sur la "toile numérique », on trouve finalement sur un résumé d'assemblée générale du Conseil des droits de l'humain des Nations Unies tenu le 5 août 2010 73, où l'on peut lire un paragraphe qui dénonce toujours le manque de femmes au sein des instances politiques (p. 9), puis une longue liste des associations et réseaux d'associations ayant contribué à la rédaction du document : les associations de femmes (et leurs nouveaux noms, telle que le Centre for Human Rights and Development, créé par une ancienne salariée de l'Association des femmes juristes) ne sont pas moins d'une quinzaine. Enfin, on note avec intérêt la création d'un réseau qui parait dynamique, le MONFEMNET 74 , et l'apparition de nouvelles thématiques de mobilisation de femmes comme la protection de la nature ${ }^{75}$. Ainsi, les femmes sont toujours bien actives aujourd'hui et évoluent même vers de nouvelles causes, dont on peut supposer qu'elles apparaissent aussi dans les propositions de nouvelles subventions d'organisations internationales. Il reste alors à observer, de concert avec les militantes et leurs attentes, comment les leaders politiques mongols vont recevoir ces nouvelles thématiques «englobantes » que sont les droits humains et la protection de la nature, au sein desquelles les femmes tentent de distiller leurs propres revendications.

72. http://www.cca.mn/

73. A/HRC/WG.6/9/MNG/3; http://upr-epu.com/files/201/Parties.pdf

74. http://www.monfemnet.org/en.php

75. Environment and Development Women Center; The Gender Center for Sustainable Development ; Nature and Women Centre for Promoting Environmental Development ; "Motherland E Family" Women association. 